\title{
Neurological consequences of recreational nitrous oxide abuse during SARS-CoV-2 pandemic
}

\author{
Raphael Vollhardt ${ }^{1}$ (1) $\cdot$ Julie Mazoyer ${ }^{1} \cdot$ Lucy Bernardaud $^{1} \cdot$ Andrei Haddad $^{1} \cdot$ Pauline Jaubert $^{1} \cdot$ Irène Coman $^{1}$. \\ Philippe Manceau ${ }^{1} \cdot$ Marie Mongin ${ }^{1} \cdot$ Bertrand Degos $^{1,2}$
}

Received: 25 May 2021 / Revised: 1 August 2021 / Accepted: 6 August 2021 / Published online: 21 August 2021

(c) Springer-Verlag GmbH Germany, part of Springer Nature 2021

\begin{abstract}
Introduction Recreational use of nitrous oxide $\left(\mathrm{N}_{2} \mathrm{O}\right)$ is a growing practice in France and all around the world and is often associated with neurological complications. We report detailed clinical and paraclinical presentations of 12 patients with combined degeneration of the spinal cord and peripheral neuropathies in relation to $\mathrm{N}_{2} \mathrm{O}$ consumption, possibly favored by lockdowns due to SARS-CoV-2 pandemic.

Results With variable levels of consumption, the 12 patients presented spinal cord and/or peripheral nerve damage, with mostly motor and ataxic symptoms, motor axonal nerve damage, and medullary T2-weighted hyperintensities on MRI. There was a clear improvement in symptoms after vitamin B12 substitution, although some sequelae remained, particularly sensory. Discussion We report detailed clinical, electrophysiological, radiological, and biological consequences of $\mathrm{N}_{2} \mathrm{O}$ abuse in 12 patients. Our data support the clinical and paraclinical observations reported in the literature. The mechanisms of neurological $\mathrm{N}_{2} \mathrm{O}$ toxicity are still debated. There is currently no precise recommendation on the therapeutic management. The clinical evolution after vitamin B12 substitution seems sufficient but could depend on early management. Effective messages targeting at risk population, but also the health professionals involved, seem crucial as does a better legal framework for this growing practice.
\end{abstract}

Keywords Nitrous oxide $\cdot$ Neuropathy $\cdot$ Myelopathy $\cdot$ Neurological disorder

\section{Introduction}

Exposure to nitrous oxide $\left(\mathrm{N}_{2} \mathrm{O}\right)$ is associated with multiple complications, especially neurological ones [1]. The first reported cases mainly concerned situations of occupational or post-operative exposure.

Recreational use of $\mathrm{N}_{2} \mathrm{O}$, mainly through freely bought food cartridges, inhaled either directly or via a balloon, has

Raphael Vollhardt and Julie Mazoyer have equally contributed to this work.

Bertrand Degos

bertrand.degos@aphp.fr

1 Service de Neurologie, APHP, Hôpital Avicenne, Hôpitaux Universitaires de Paris - Seine Saint Denis, Sorbonne Paris Nord, Bobigny, France

2 Dynamics and Pathophysiology of Neuronal Networks Team, Center for Interdisciplinary Research in Biology, Collège de France, CNRS, UMR7241/INSERM U1050, Université PSL, 75005 Paris, France sharply increased since a few years. It was believed to be the second most common psychoactive substance consumed by French students between 2015 and 2017 [2]. Since then, there has been a growing frequency of serious complications linked to this consumption, particularly in France, as indicated in reports by the French addictovigilance network and the French Health Security Agency in June 2020. Surveillance data on psychoactive substances consumed in France identify an important and sustained $\mathrm{N}_{2} \mathrm{O}$ consumption during the SARS-CoV-2 epidemic, especially during the first lockdown, in relation to a difficulty of access to other substances and a need to consume due to inactivity [3]. Worldwide, $\mathrm{N}_{2} \mathrm{O}$ abuse is already responsible for several cases of death [1].

In our article, we describe clinically, biologically, radiologically and neurophysiologically 12 cases of spinal cord injury and/or peripheral neuropathies secondary to $\mathrm{N}_{2} \mathrm{O}$ recreational consumption that occurred between August 2020 and April 2021 and were treated in our Neurology 
Department at the Avicenne University Hospital. All the data were obtained from the records of patients.

\section{Results}

The clinical and paraclinical data from all 12 patients are detailed in Table 1. There were 6 males and 6 females with an average age of $22.2 \pm 3.3$ years. The mean weekly consumption of $\mathrm{N}_{2} \mathrm{O}$ was of $3109 \pm 2800 \mathrm{~g}$ with a mean duration of $12.7 \pm 8.3$ months between the onset of consumption and the hospitalization. Ten patients were regular smokers (cigarette or Hookah), three were occasional cannabis users, and seven were alcohol users (only one in a daily basis). None of them was occupationally exposed to heavy metals, nitrogen protoxide, or industrial solvents. Four of them were unemployed or out of school, one was a student, and the others worked as animators, cleaning lady or delivery drivers.

All of the patients presented impaired vibratory sensation or proprioceptive ataxia of lower limbs (12/12) and frequent motor deficits (9/12) predominantly in the lower limbs. Seven patients had spinal cord injury symptoms (pyramidal reflexes, Lhermitte sign, vesico-sphincterian disorders, sensitive spine level). Other neurologic manifestations included dysexecutive syndrome (1/12) and visual disturbance (1/12). Two patients experienced paranoia, one of them (patient 2) requiring a psychiatric hospitalization for a first and transitory psychotic disorder, responsible for a delay in B12 supplementation. As shown in Fig. 1, every patient with an important $\mathrm{N}_{2} \mathrm{O}$ consumption ( $>5000 \mathrm{~g} /$ week) had a Rankin scale $\geq 3$. However, the small number of included patients did not allow us to highlight a statistically significant association.

Electroneuromyograms showed a neuropathy in 10/12 patients, with typical axonal and motor damage, predominantly in the lower limbs. One presented a demyelinating neuropathy, another initially presented a normal electroneuromyography, but two months later (after supplementation) displayed a pure motor impairment of the lower limbs. Axonal loss was predominant on peroneal nerves with no apparent relationship with $\mathrm{N}_{2} \mathrm{O}$ consumption. Among the 3 patients with an absence of motor response on peroneal nerves, 2 had a consumption lower than the median.

MRI of the spinal cord showed extensive T2-weighted hyperintensities in 9/12 patients, with a stereotypical involvement of posterior cords, and cervical predominance. One of the patients with normal spinal cord MRI had the highest $\mathrm{N}_{2} \mathrm{O}$ consumption. Two patients (patients 1 and 6) presented gadolinium enhancement of the posterior cervical cord, one of which was also meningeal (patient 6). MRI control at 2 and 4 weeks, in patients 1 and 6 , respectively, showed a stability of the T2-weighted hyperintensities but an improvement in the medullary and meningeal enhancement.
Cerebrospinal fluid analyses performed in those two subjects and another patient showed no inflammatory, no oligoclonal bands, and no viral abnormalities. Metabolic, infectious (e.g., HIV, syphilis), and inflammatory tests were performed and were all negative except for a folate deficiency found in three subjects, and a selenium, zinc, and iron deficiency in one patient. As the clinical presentations were all acute or subacute, no genetic testing was performed. In addition, no patient reported a family history of neurological disorders and they all started to clinically improve after $\mathrm{N}_{2} \mathrm{O}$ weaning and vitamin supplementation.

All our patients benefited from intramuscular (10/12) or oral (2/12) B12 supplementation, but one did not wish to continue treatment (patient 2). Symptoms stabilized in all of them consecutive to $\mathrm{N}_{2} \mathrm{O}$ withdrawal and $\mathrm{B} 12$ vitamin substitution. Long-term follow-up was mostly difficult, with poor adherence to care and patients were frequently lost to follow-up. Eight out of twelve patients have been re-evaluated at a mean time of $7.6 \pm 6.9$ weeks after supplementation. These patients notably improved with a Rankin score variation of $1.7 \pm 1.0$, but all of them displayed persistent motor or sensory symptoms at last visit.

\section{Discussion}

Here, we described 12 cases of patients developing neurological complications with spinal cord and peripheral nerve involvement secondary to $\mathrm{N}_{2} \mathrm{O}$ abuse during the SARS$\mathrm{CoV}-2$ pandemic. Importantly, their clinical and paraclinical conditions improved after B12 supplementation.

Since the summer of 2020, the resurgence of such neurological complications in our Parisian suburb of Seine-Saint Denis Department suggests that the SARS-CoV-2 pandemic with its successive lockdowns may have favored an increased consumption of $\mathrm{N}_{2} \mathrm{O}$. Indeed, at least 7 of our 12 patients started their consumption in 2020 or 2021 , mostly exceeding the framework of a simply social and "festive" consumption.

The clinical presentations of our patients are comparable to that of cases previously described in the literature $[1,4$, 5]. Peripheral nerve damage seems to be at least as frequent as spinal cord damage, with essentially motor, sensory, and ataxic symptoms. The peripheral neuropathy is responsible for a dramatic impairment of autonomy as is the case in our patients, three of them having a modified Rankin score of 4 . Although not frequent, we also report the development of psychiatric disorders.

Electroneuromyographic studies described in previous reports showed a high prevalence of motor axonal damages as is the case with our patients, but sometimes results were more heterogeneous, mainly highlighting demyelinating polyneuropathies $[4,6]$. 


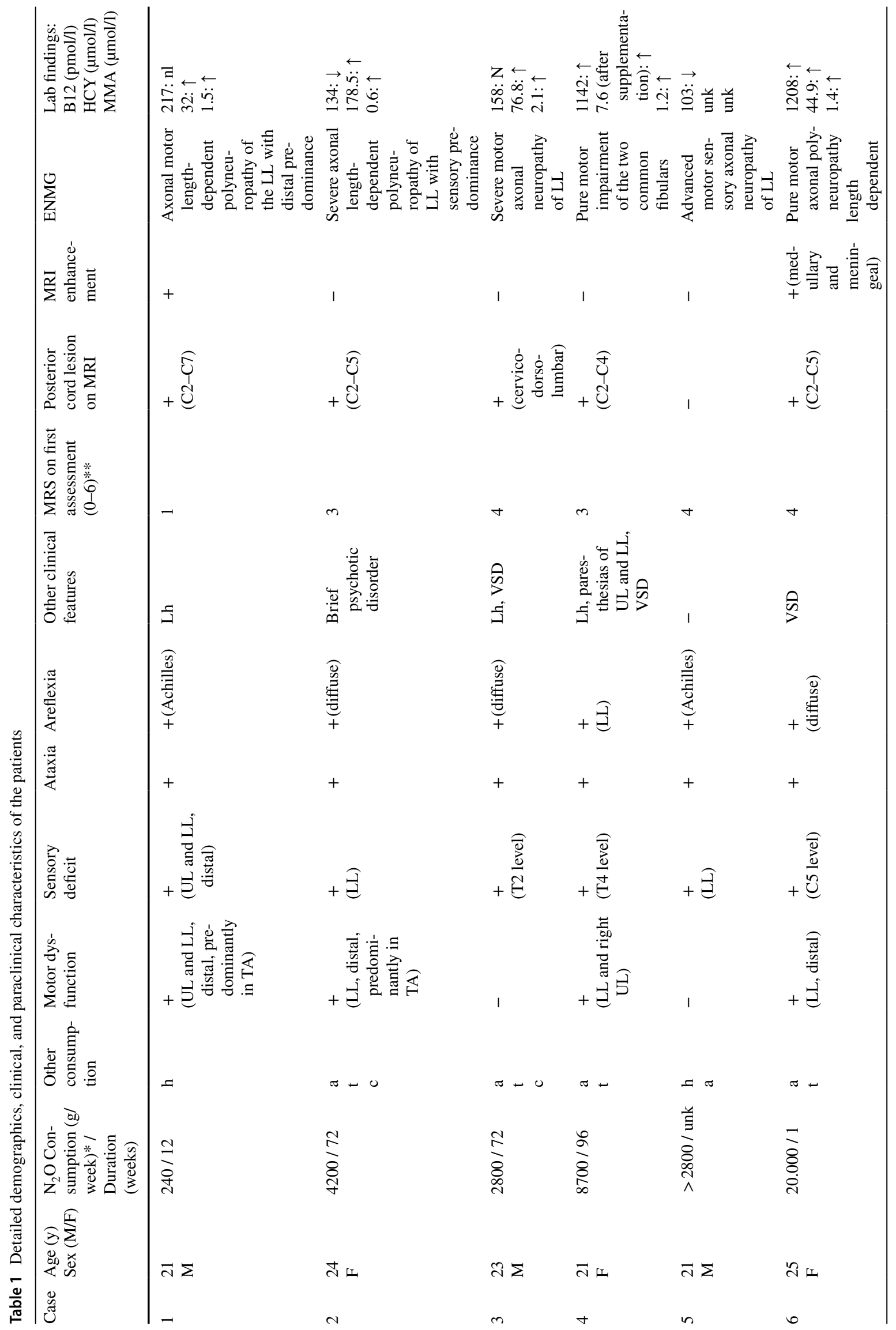




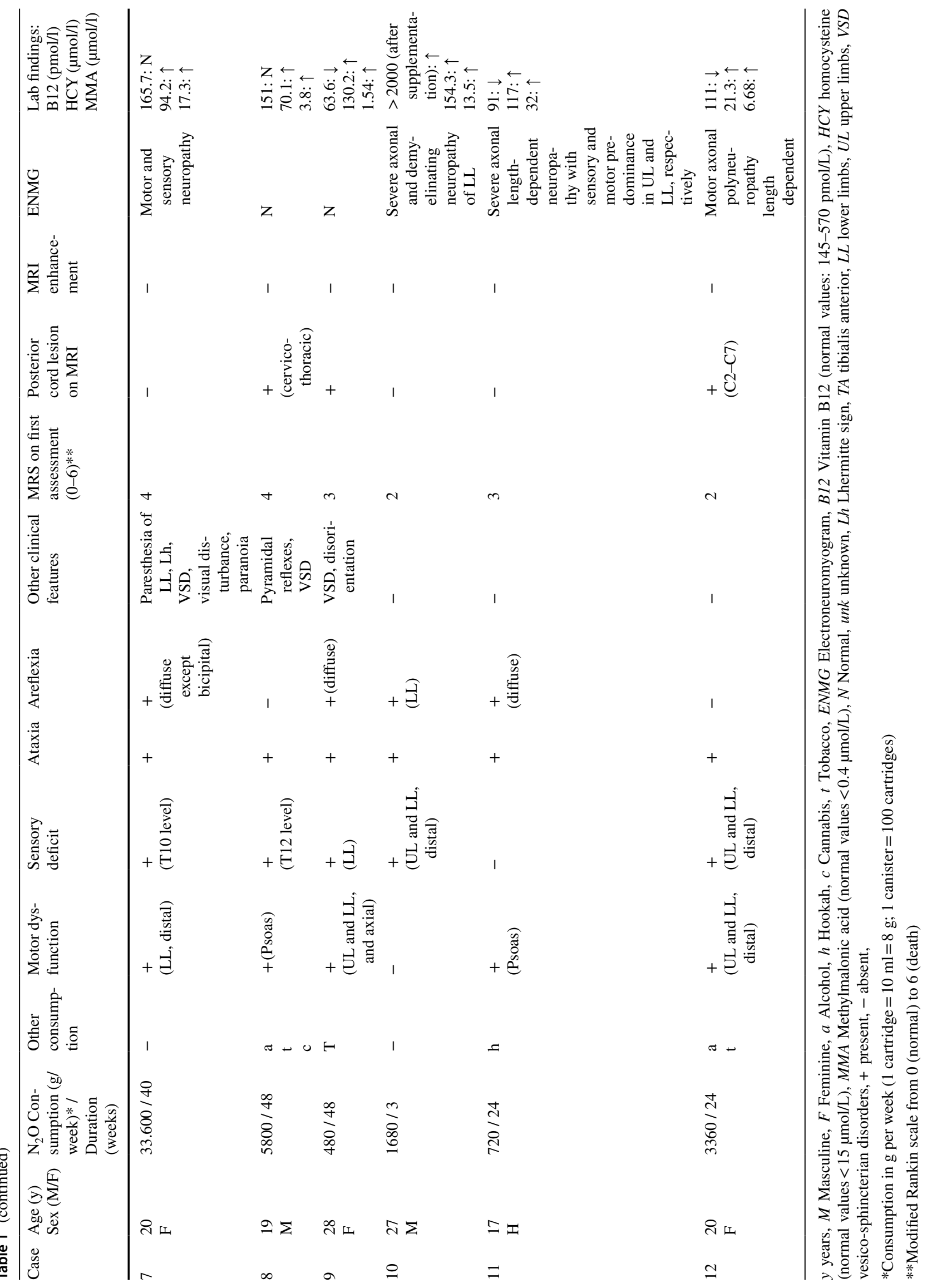


Fig. 1 Relation between nitrous oxide consumption and modified Rankin scale on first assessment. MRS modified Rankin scale. *Consumption in gram per week $(1$ cartridge $=10 \mathrm{ml}=8 \mathrm{~g} ; 1$ canister $=100$ cartridges)

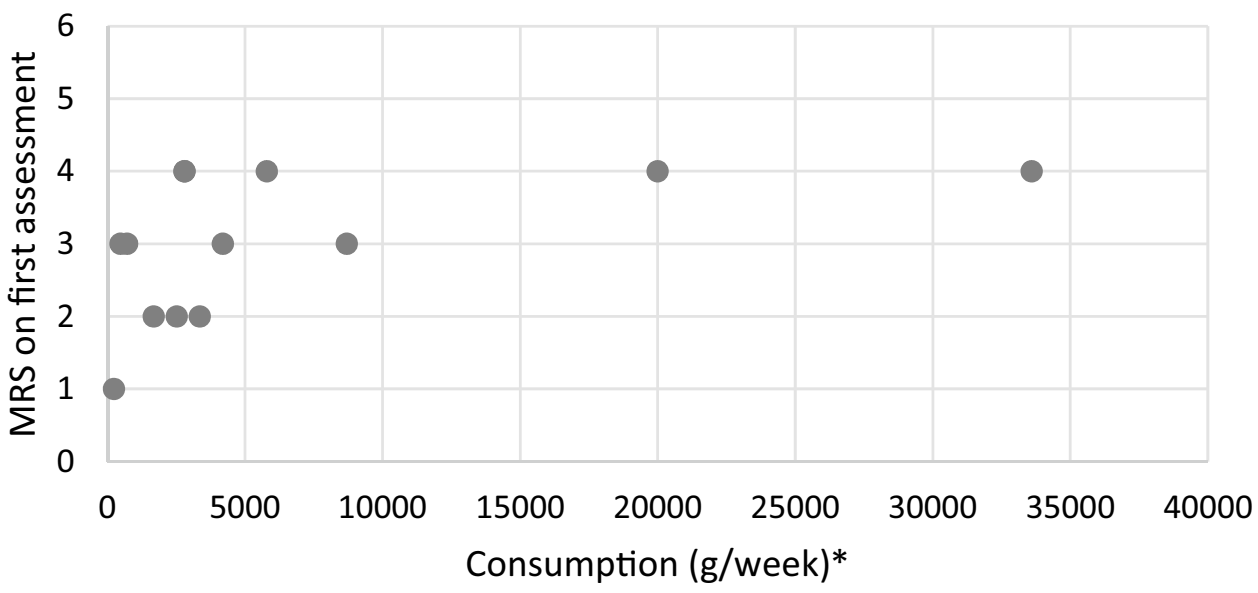

T2-weighted hyperintensities on spinal cord MRI are usually stereotyped, essentially posterior, symmetrical, often extensive, and predominantly cervical, with a reported prevalence of $68 \%$ to $78 \%$ depending on the studies [1, 4]. None of our four patients with brain MRI had any abnormalities; these have rarely been described and appear to be aspecific. Surprisingly, 2/12 patients showed spinal cord contrast enhancement which has only been rarely described [7]. To our knowledge, no cases of meningeal contrast enhancement have been previously reported. Although these patients presented a typical clinico-radiological disorder with clear improvement after $\mathrm{N}_{2} \mathrm{O}$ withdrawal and $\mathrm{B} 12$ vitamin substitution, the hypothesis of an underlying or associated inflammatory disorder remains possible. In such cases, prolonged clinical and radiological follow-up appears essential.

The most sensitive biological markers of these $\mathrm{N}_{2} \mathrm{O}$-related disorders in the literature seem to be an increase in methylmalonic acid and homocysteine levels [4] Vitamin B12 levels are often below normal limits, although the deficit might be, at least initially, only functional. The assays performed in our patients are consistent with these observations. A control, carried out in 2 of our patients, one month after supplementation showed a normalization of methylmalonic acid and homocysteine levels.

There is no consensus on the mode, dose, and minimum duration of vitamin B12 supplementation. Although essentially intramuscular, some authors propose an oral supplementation by analogy with the management of pernicious anemia where high daily oral doses (1000-2000 $\mu \mathrm{g} / \mathrm{d}$ ) could bring similar results [8]. Interestingly, a myeloprotective efficacy of methionine has been demonstrated in certain animal models of vitamin B12 deficiency [9] and although no controlled studies have been carried out in humans, some authors propose this molecule as a complement to vitamin substitution.

Among the cases reported in the literature, follow-up data, when available, show that the clinical course is mostly favorable, with partial if not complete recovery [1]. This is similar to supplemented cases of combined marrow sclerosis due to vitamin B12 deficiency for which long-term sequelae remain in only $6 \%$ of patients [10]. However, some authors point to a higher rate of sequelae $[11,12]$. Early vitamin B12 supplementation and $\mathrm{N}_{2} \mathrm{O}$ withdrawal seem to play a determining role in this evolution $[10,12]$.

A threshold for toxic consumption seems difficult to determine, because of the wide variety of consumption patterns and modes, and because it is likely that this threshold varies between subjects depending on different factors [13]. However, a probability model has recently been proposed, illustrating a dose-response relationship between the number of cartridges consumed per session and the occurrence of paresthesia [14].

Several pathophysiological hypotheses have been proposed to explain the occurrence of $\mathrm{N}_{2} \mathrm{O}$ complications and involve the inactivation of vitamin $\mathrm{B} 12$ by oxidation of the cobalt atom it contains. Two pathways resulting from the active forms of this vitamin in the human body (AdoB12 and MetB12) were implicated to explain those complications: Either from an imbalance of fatty acids and by extension of myelin synthesis or via a decrease in the production of S-adenosyl methionine, a universal $\mathrm{CH} 3$ donor, which would play an important role in the synthesis of myelin binding protein and myelin lipids. More recently, it has been proposed that myelopathy lesions could result from a disturbance in the cytokine balance between TNF alpha and IL6/ IL10, with S-adenosyl methionine appearing to be involved in their production, and their myelotoxic or myeloprotective roles having, respectively, been demonstrated [15].

Although our study is limited by its retrospective feature, we reported numerous cases of neurological $\mathrm{N}_{2} \mathrm{O}$-induced complications. Further prospective studies are needed notably to determine which treatment regimen is preferable and to assess the long-term improvement by clinical examination, biological tests, ENMG, and medullary MRI.

Regarding the increasing frequency of $\mathrm{N}_{2} \mathrm{O}$ abuse and its potential neurological sequelae, the importance of effective 
public health messages targeting at-risk populations and of information for the health professionals who have to deal with them seems crucial, as should be a better legal framework for this practice.

Acknowledgements The authors thank Sébastien Valverde for English editing

Funding This study was not supported by any funding.

Data availability The authors confirm that the data supporting the findings of this study are available within the article.

Code availability Not applicable.

\section{Declarations}

Conflicts of interest The authors declare that they have no competing interests.

Ethical approval The current study obtained the authorization of the Local Ethics Committee of Avicenne Hospital (CLEA-2021-200).

Consent to participate Written consents were obtained from all participants.

Consent for publication Written consents were obtained from all participants.

\section{References}

1. Garakani G, Jaffe RJ, Salva D et al (2016) Neurologic, psychiatric, and other medical manifestations of nitrous oxide abuse: a systematic review of the case literature. Am J Addict. https://doi. org/10.1111/ajad.12372

2. Perino J, Letinier L, Mathieu C et al (2018) Consommation de substances psychoactives : un état des lieux au sein des étudiants de la cohorte i-Share. Ther Recreat J 73:575. https://doi.org/10. 1016/j.therap.2018.09.019

3. Lapeyre-Mestre M, Boucher A, Daveluy A et al (2020) Addictovigilance contribution during COVID-19 epidemic and lockdown in France. Therapie 75:343-354. https://doi.org/10.1016/j. therap.2020.06.006
4. Oussalah A, Julien M, Levy J et al (2019) Global burden related to nitrous oxide exposure in medical and recreational settings: a systematic review and individual patient data meta-analysis. J Clin Med. https://doi.org/10.3390/jcm8040551

5. Zheng D, Ba F, Bi G et al (2020) The sharp rise of neurological disorders associated with recreational nitrous oxide use in China: a single-center experience and a brief review of Chinese literature. J Neurol 267:422-429. https://doi.org/10.1007/ s00415-019-09600-w

6. Tani J, Weng H-Y, Chen H-J et al (2019) Elucidating unique axonal dysfunction between nitrous oxide abuse and vitamin B12 deficiency. Front Neurol 10:704. https://doi.org/10.3389/fneur. 2019.00704

7. Ernst LD, Brock K, Barraza LH et al (2015) Longitudinally extensive nitrous oxide myelopathy with novel radiographic features. JAMA Neurol 72:1370-1371. https://doi.org/10.1001/jamaneurol. 2015.2141

8. Stabler SP (2013) Clinical practice. Vitamin B12 deficiency. N Engl J Med 368:149-160. https://doi.org/10.1056/NEJMcp1113 996

9. Scott JM, Dinn JJ, Wilson P, Weir DG (1981) Pathogenesis of subacute combined degeneration: a result of methyl group deficiency. Lancet 2:334-337. https://doi.org/10.1016/s01406736(81)90649-8

10. Healton EB, Savage DG, Brust JC et al (1991) Neurologic aspects of cobalamin deficiency. Medicine (Baltimore) 70:229-245. https://doi.org/10.1097/00005792-199107000-00001

11. Thompson AG, Leite MI, Lunn MP, Bennett DLH (2015) Whippits, nitrous oxide and the dangers of legal highs. Pract Neurol 15:207-209. https://doi.org/10.1136/practneurol-2014-001071

12. Vasconcelos OM, Poehm EH, McCarter RJ et al (2006) Potential outcome factors in subacute combined degeneration: review of observational studies. J Gen Intern Med 21:1063-1068. https:// doi.org/10.1111/j.1525-1497.2006.00525.x

13. Lacassie HJ, Nazar C, Yonish B et al (2006) Reversible nitrous oxide myelopathy and a polymorphism in the gene encoding 5,10-methylenetetrahydrofolate reductase. Br J Anaesth 96:222225. https://doi.org/10.1093/bja/aei300

14. Winstock AR, Ferris JA (2020) Nitrous oxide causes peripheral neuropathy in a dose dependent manner among recreational users. J Psychopharmacol 34:229-236. https://doi.org/10.1177/02698 81119882532

15. Hathout L, El-Saden S (2011) Nitrous oxide-induced $B_{12}$ deficiency myelopathy: perspectives on the clinical biochemistry of vitamin $B_{12}$. J Neurol Sci 301:1-8. https://doi.org/10.1016/j.jns. 2010.10.033 